\title{
Spectral properties of the small polaron
}

\author{
J. M. Robin \\ Max-Planck-Institut für Physik komplexer Systeme, Bayreuther Straße 40 Haus 16, 01187 Dresden, Germany
}

(January 23, 2014, e-mail: robin@mpipks-dresden.mpg.de)

\begin{abstract}
We compute exactly both the spectral function of the electron and of the small polaron for the two site Holstein model. We find that for intermediary coupling, the small polaron is a better fundamental excitation of the system than the electron. However, the Lang-Firsov approximation fails to predict the right dispersion relation for the small polaron.
\end{abstract}

Electronic properties of strongly interacting electronphonon systems are now widely studied since it has been realized experimently that the formation of small polarons in some of the new materials, such as the high $T_{c}$ cuprates [1] or the perovskite manganates [2], is responsible in part of some new and unusual physics.

It is generally believed that the simplest model for the study of small polarons, is the so called Holstein molecular crystal model [3]. In the weak coupling limit, electrons behave as good quasiparticles, while in the strong coupling limit the model is believed to exhibit well defined small polarons [4]. Perturbation theory towards intermediary coupling can then be used from the strong limit fixed point in term of small polaron quasiparticles [4.11]. The validity of such a perturbation theory rely on the quality of the small polaron quasiparticle. If it is destroyed away from infinite coupling then we can no longer described the physics in term of small polarons and no longer use perturbation theory.

In this paper, we compute exactly the spectral functions for the small polarons in two-site Holstein model for intermediary coupling. We find that they are good quasiparticles with a well defined relation dispersion $\omega_{k}^{*}$. We can then compute the effective bandwidth of the small polarons $D^{*}=\max \left(\omega_{k}^{*}\right)-\min \left(\omega_{k}^{*}\right)$ and show that it dramatically decreases with the strength of the electronphonon coupling constant. These results clearly show that the physical picture given by Lang and Firsov is the right one.

Our calculations are based on a two site cluster where exact diagonalization are easily performed. The hamiltonian for the Holstein model is

$$
\begin{aligned}
H & =-t \sum_{j, \delta, \sigma} c_{j+\delta, \sigma}^{\dagger} c_{j, \sigma}+\omega_{0} \sum_{j} b_{j}^{\dagger} b_{j} \\
& -g \omega_{0} \sum_{j, \sigma} c_{j, \sigma}^{\dagger} c_{j, \sigma}\left(b_{j}^{\dagger}+b_{j}\right) .
\end{aligned}
$$

The sum over $\delta$ run on the nearest neighbours, $t$ is the hopping integral for the tight binding approximation, $\omega_{0}$ is the optical frequency of the phonon and $g$ is a dimensionless coupling constant. The polaronic energy is $E_{P}=g^{2} \omega_{0}$ and the usual electron-phonon coupling constant is $\lambda=E_{P} / z t$ where $z$ is the number of nearest neighbours ( $z=1$ for the two site cluster).
In the atomic limit, $t=0$, the hamiltonian can be diagonalized exactly by the Lang-Firsov transformation [5]. The new hamiltonian is $\tilde{H}=e^{-S} H e^{S}$ with $S=$ $\sum_{j} g\left(b_{j}^{\dagger}-b_{j}\right) n_{j}$ and $n_{j}=\sum_{\sigma} c_{j, \sigma}^{\dagger} c_{j, \sigma}$. We thus obtain $\tilde{c}_{j, \sigma}=U_{j} c_{j, \sigma}$ and $\tilde{b}_{j}=b_{j}+g n_{j}$ with

$$
U_{j}=e^{g\left(b_{j}^{\dagger}-b_{j}\right)} .
$$

The transformed hamiltonian is

$$
\begin{aligned}
\tilde{H}= & -t \sum_{j, \delta, \sigma} c_{j+\delta, \sigma}^{\dagger} U_{j+\delta}^{\dagger} U_{j} c_{j, \sigma}-E_{P} \sum_{j} n_{j} \\
& +\omega_{0} \sum_{j} b_{j}^{\dagger} b_{j}-2 E_{P} \sum_{j} n_{j, \uparrow} n_{j, \downarrow} .
\end{aligned}
$$

The hopping term is now a complicated operator. In the Lang-Firsov approximation, one obtains an effective hamiltonian for the electrons by eliminating the phonon states, keeping only the vacuum without phonon. At zero temperature one gets $U_{j} \rightarrow e^{-g^{2} / 2}$ and thus an effective hopping integral $t^{*}=e^{-g^{2}} t$ for the small polaron. In the strong coupling limit, $g \rightarrow \infty$, this approximation gives localised polarons, while for finite coupling it gives a polaronic band with the dispersion relation $\varepsilon_{\mathbf{k}}^{*}=-E_{P}+t^{*} \xi_{\mathbf{k}}$, where $\xi_{\mathbf{k}}$ is the Fourier transform of the kinetic term. For the two site cluster, one gets $\varepsilon_{k}^{*}=-E_{P} \pm t^{*}$ for $k=0, \pi$. If one considers systems with less than two electrons, then the interaction term responsible of the formation of bipolarons (the last term of eq.(3)) vanishes and the small polarons energies $\varepsilon_{\mathbf{k}}^{*}$ are the right excitations of the system. The spectral function of a small polaron, always in the Lang-Firsov approximation, is simply $A(\mathbf{k}, \omega)=2 \pi \delta\left(\omega-\varepsilon_{\mathbf{k}}^{*}\right)$. Inversely, the electrons are no longer good excitations. They carry a cloud of phonons, with an average number of phonon $n g^{2}$, where $n$ is the number of electron. The spectral function is given by [5, 6 ]

$$
\begin{gathered}
A(\mathbf{k}, \omega)=e^{-g^{2}} 2 \pi \delta\left(\omega-\varepsilon_{\mathbf{k}}^{*}\right) \\
+e^{-g^{2}} \frac{1}{M} \sum_{\mathbf{k}^{\prime}} \sum_{\ell=1}^{\infty} \frac{g^{2 \ell}}{\ell !} 2 \pi \delta\left(\omega-\varepsilon_{\mathbf{k}^{\prime}}^{*}-\ell \omega_{0}\right),
\end{gathered}
$$

where $M$ is the number of sites. Exact diagonalizations of small clusters [7] based on the bare hamiltonian of eq.(1) showed that the spectral function for the electron is too 
much complicated to be interpreted (as can be seen in fig.11). For intermediary and strong coupling, there is no longer a quasiparticle peak and thus impossible to extract a dispersion relation. One possible test for the validity of the Lang-Firsov approximation should be to fit the numerical results for the spectral function of the electron with the formula of eq.(位). A more efficient method consist to compute directly the spectral function of the small polaron based on the transformed hamiltonian of eq.(3). If the small polaron defined by the canonical transformation is a good quasiparticle then the spectral function should contains only one main peak with a width smaller than the excitation energy. It is then easier to compare this energy to the one predicts by the Lang-Firsov approximation. However the aim of this paper is simply to set up if the small polaron is a good quasiparticle for the system.

In the transformed hamiltonian $\tilde{H}$, the operators $c_{j, \sigma}$ and $U_{j}$ commute. One can build separately the operator $c_{j+\delta, \sigma}^{\dagger} c_{j, \sigma}$ and then work in a subspace with a fixed number of polarons. The operator $U_{j}$ is computed via its matrix elements given by

$U_{n^{\prime}, n}=e^{-g^{2}} \sum_{p=0}^{n} \sum_{p^{\prime}=0}^{n^{\prime}}(-)^{p} \frac{g^{\left(p+p^{\prime}\right)}}{p ! p^{\prime} !}\left[\frac{n ! n^{\prime} !}{(n-p) !\left(n^{\prime}-p^{\prime}\right) !}\right]^{1 / 2}$

and the condition $n-p=n^{\prime}-p^{\prime}$. For the phonon basis states, a better convergence is obtained if we define some subspaces with constant total number of phonons [8]. We diagonalize matrices with a maximum of 35 phonons (corresponding to 666 basis states of phonons). Comparison of the eigenvalues of both the bare and transformed hamiltonians $H$ and $\tilde{H}$ shows that the polaronic representation is better for the results presented here. In order to deal with the case $\omega_{0} \simeq t$, we choose the same parameters as in ref. [9] where the spectral function $J(k, \omega)$ for the electron has been computed, that is $\omega_{0}=1 / 1.1$, $g=0.3 \sqrt{2}, 0.8 \sqrt{2}$ and $1.3 \sqrt{2}$. We compute the spectral function at zero temperature in the ground state with zero small polaron defined by

$$
\begin{aligned}
\tilde{J}(k, \omega)= & 2 \pi \sum_{m}\left|\left\langle N=1, m\left|c_{k, \uparrow}^{\dagger}\right| N=0,0\right\rangle\right|^{2} \\
& \times \delta\left(\omega+E_{0}^{N=0}-E_{m}^{N=1}\right)
\end{aligned}
$$

The number of phonon states has been chosen such that the results presented here are well converged. The criterium is that the transition amplitudes and energies converge towards a finite limit as we increase the number of phonon states.
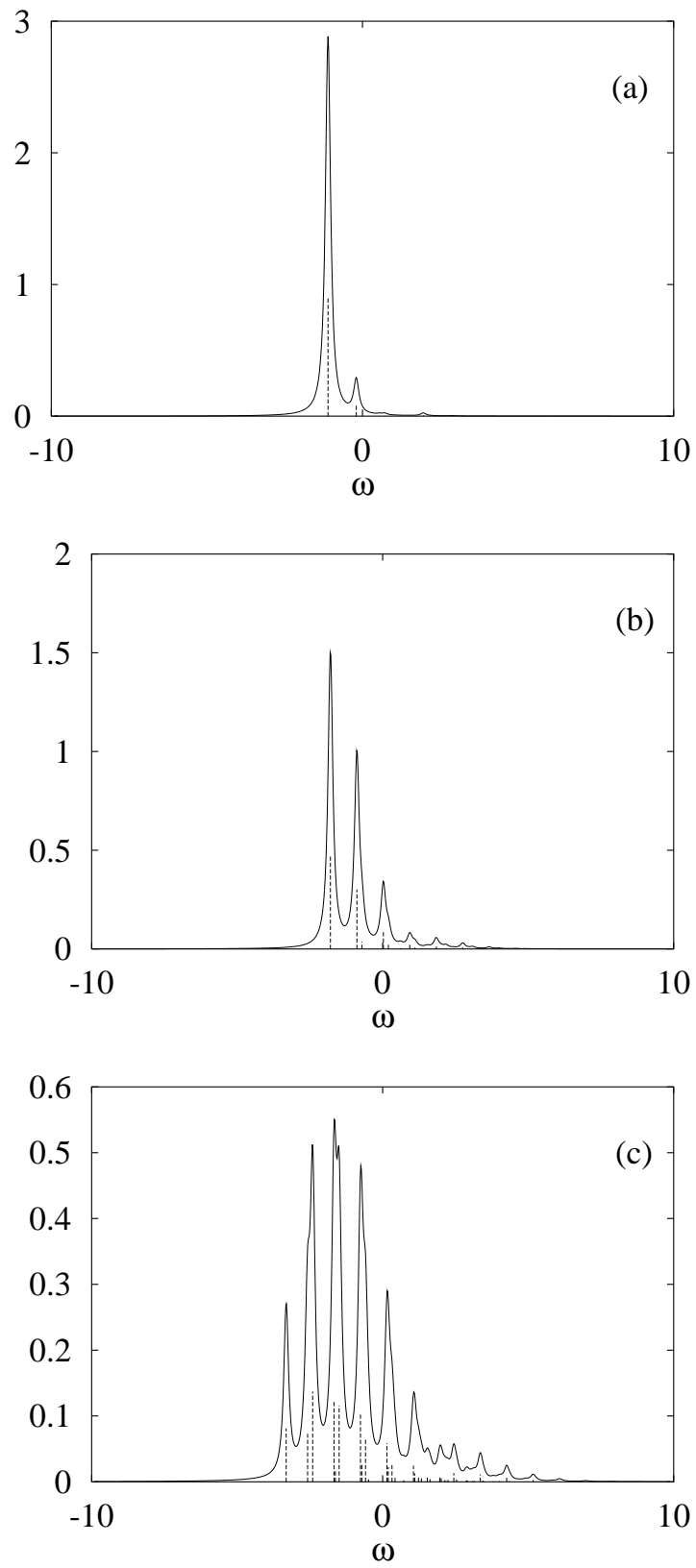

FIG. 1. Spectral functions $J(k=0, \omega)$ for the electron, for three different couplings and $\omega_{0}=1 / 1.1, g=0.3 \sqrt{2}$ (a), $g=0.8 \sqrt{2}(\mathrm{~b}), g=1.3 \sqrt{2}$ (c).

In fig.11, we show the spectral functions of the electron based on the hamiltonian of eq.(11) which correspond with the results of ref. [9] (we note that these are computed using two different methods) and in fig.2 2 we show the spectral functions of the small polaron based on the hamiltonian of eq.(3). The solid lines in figures correspond to Lorentzian of width $0.1 t$ while peaks correspond to the value of the transition amplitude, without the $2 \pi$ factor. We see that for weak enough coupling, both the small polaron and the electron are good quasiparticle with mainly one transition in the spectral function. As we increase the coupling we observe for the small polaron 
that the spectral weight stays in one peak while it spreads in many peaks more or less spaced of the energy $\omega_{0}$ for the electron. For $g=0.3 \sqrt{2}$ we find $\omega_{k=0} \simeq-1.108$ and $\omega_{k=\pi} \simeq 0.977$, for $g=0.8 \sqrt{2}, \omega_{k=0} \simeq-1.795$ and $\omega_{k=\pi} \simeq-1.349$, and for $g=1.3 \sqrt{2}, \omega_{k=0} \simeq-3.314$ and $\omega_{k=\pi} \simeq-3.225$. All the results are in unit of $t$.
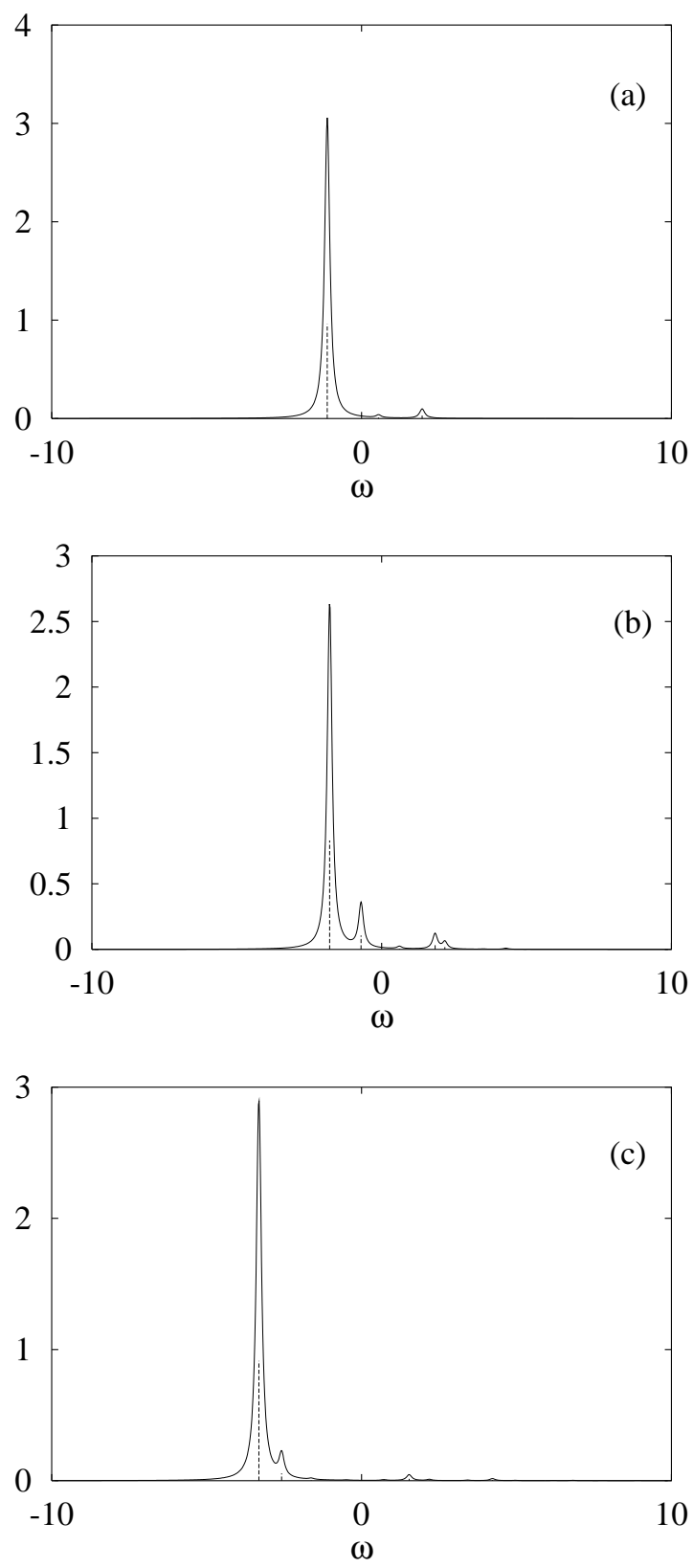

FIG. 2. Spectral functions $\tilde{J}(k=0, \omega)$ for the small polaron, for three different couplings and $\omega_{0}=1 / 1.1, g=0.3 \sqrt{2}$ (a), $g=0.8 \sqrt{2}(\mathrm{~b}), g=1.3 \sqrt{2}(\mathrm{c})$.

If we compare the renormalized bandwidth $D^{*}=$ $\omega_{k=\pi}-\omega_{k=0}$ with the Lang-Firsov approximation $D_{L F}^{*}=$ $2 t e^{-g^{2}}$ we find a discrepancy of $25 \%$ for the two strongest couplings.

To conclude, the approach presented here is different from the standard approach of the so-called polaron problem where one considers a system with only one electron coupled to the lattice and find the excitation energy of the whole Hamiltonian [10]. Instead, we have shown that the small polaron defined through the canonical transformation of Lang-Firsov is a good quasiparticle for strong and intermediary electron-phonon coupling. This method applies directly to many body problems, such as the Hamiltonian of eq.(3) for the Holstein model and for arbitrary filling of the system. Perturbative expansion from e.g. the strong coupling Lang-Firsov approximation fixed point [4.11] should be relevant.

\section{ACKNOWLEDGMENTS}

I would like to thank D. Devillers, M. van den Bossche, F. Vistulo de Abreu, H. Geoffray and M. S. Laad for interesting discussions. Part of the numerical calculations were performed at Centre de Recherche sur les Très Basses Températures (CRTBT), Grenoble.

[1] Lattice Effects in High Temperature Superconductors, ed. Y. Bar-Yam, J. Mustre de Leon and A. R. Bishop (World Scientific, Singapore, 1992).

[2] A. J. Millis, B. I. Schraiman and R. Mueller, Phys. Rev. Lett. 77, 175 (1996); H. Röder, J. Zang and A. R. Bishop, Phys. Rev. Lett. 76, 1356 (1996).

[3] T. Holstein, Ann. Phys. (NY) 8, 325 (1959).

[4] I. J. Lang and Yu. A. Firsov, Soviet Physics JETP 16, 1301 (1962).

[5] G. D. Mahan, Many-Particle Physics, Plenum Press, (New York, London 1990).

[6] A. S. Alexandrov and J. Ranninger, Phys. Rev. B 45, 13109 (1992).

[7] J. Ranninger and U. Thibblin, Phys. Rev. B 45, 395 (1992); A. S. Alexandrov, V. V. Kabanov and D. K. Ray, Phys. Rev. B 49, 9915 (1994);

[8] G. Wellein, H. Röder and H. Fehske, Phys. Rev. B 53, 9666 (1996).

[9] E. V. L. de Mello and J. Ranninger, preprint condmat/9703121

[10] R. P. Feynman, Statistical mechanics, Benjamin Reading (1972).

[11] W. Stephan, Phys. Rev. B 54, 8981 (1996). 